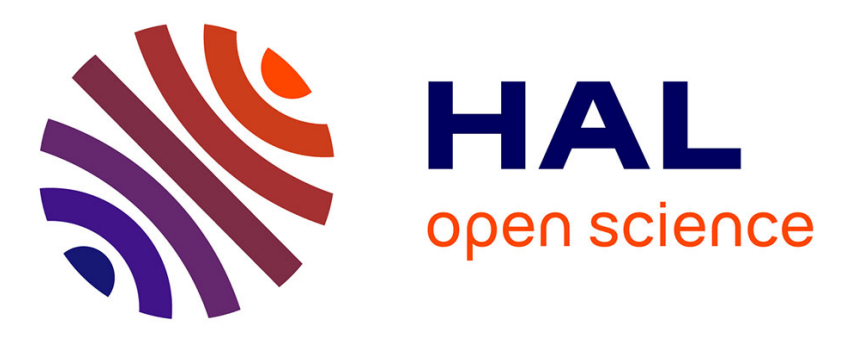

\title{
Simulation of Liquids with the Tight-Binding Density-Functional Approach and Improved Atomic Charges
}

Jérôme Cuny, Jesus C. Calatayud, Narjes Ansari, Ali A. Hassanali, Mathias Rapacioli, Aude Simon

\section{To cite this version:}

Jérôme Cuny, Jesus C. Calatayud, Narjes Ansari, Ali A. Hassanali, Mathias Rapacioli, et al.. Simulation of Liquids with the Tight-Binding Density-Functional Approach and Improved Atomic Charges. Journal of Physical Chemistry B, 2020, 124 (34), pp.7421-7432. 10.1021/acs.jpcb.0c04167 . hal02959734

\section{HAL Id: hal-02959734 \\ https://hal.science/hal-02959734}

Submitted on 6 Nov 2020

HAL is a multi-disciplinary open access archive for the deposit and dissemination of scientific research documents, whether they are published or not. The documents may come from teaching and research institutions in France or abroad, or from public or private research centers.
L'archive ouverte pluridisciplinaire HAL, est destinée au dépôt et à la diffusion de documents scientifiques de niveau recherche, publiés ou non, émanant des établissements d'enseignement et de recherche français ou étrangers, des laboratoires publics ou privés. 


\section{Simulation of Liquids with the Tight-Binding}

Density-Functional Approach and Improved Atomic Charges

Jérôme Cuny, ${ }^{*, \dagger}$ Jesus Cerda Calatayud ${ }^{\dagger}{ }^{\dagger}$ Narjes Ansari,,$\uparrow$ Ali A. Hassanali, ${ }^{\ddagger}$ Mathias Rapacioli, ${ }^{\dagger}$ and Aude Simon ${ }^{\dagger}$

$\dagger$ Laboratoire de Chimie et Physique Quantiques (LCPQ), Université de Toulouse III [UPS] and CNRS, 118 Route de Narbonne, F-31062 Toulouse, France

$\ddagger$ The Abdus Salam International Center for Theoretical Physics, Condensed Matter and Statistical Physics Section, Strada Costiera 11, 34151, Italy.

\Department of Chemistry and Applied Biosciences, ETH Zurich, 3) c/o USI Campus, Via Giuseppe Buffi13, Lugano 6900, Switzerland.

E-mail: jerome.cuny@irsamc.ups-tlse.fr

Phone: +33 (0) 561556836. Fax: +33 (0) 561556065 


\begin{abstract}
Theoretical description of liquids, especially liquid water, is an ongoing subject with important implications in various domains such as homogeneous catalysis, solvation of molecular, ionic and biomolecular species and reactivity. Various formalisms exist to describe liquids, each one displaying its own balance between accuracy and computational cost that defines its range of applications. The present article revisites the ability of the density-functional based tight-binding (SCC-DFTB) approach to model liquids by focusing on liquid water and liquid benzene under ambient conditions. To do so, we benchmark a recent correction for the SCC-DFTB atomic charges which allows for a drastic improvement of the pair radial distribution functions of liquid water as compared to both experimental data and density functional theory results performed in the generalized-gradient approximation. We also report the coupling of the deMonNano and i-PI codes to perform path-integral molecular dynamics. This allows us to rationalize the impact of nuclear quantum effects on the SCC-DFTB description of liquid water. This study evidences the rather good ability of SCC-DFTB to describe liquid water and liquid benzene. As a first example of application, we also present results for a benzene molecule solvated in water with the perspectives of further studies devoted to solvent/water interfaces.
\end{abstract}

\title{
Introduction
}

Molecular liquids are complex systems which theoretical description is of paramount importance to complement experiments in order to provide deeper insights into their structural, dynamical and thermodynamical properties. In particular, liquid water displays a variety of anomalous behaviours which make it a fascinating substance that has motivated a consider-

able amount of studies, ${ }^{1-7}$ and in particular, theoretical investigations. ${ }^{2-14}$ However, despite continuous theoretical and methodological developments in the field, the simulation of liquid water is still challenging and raises a number of theoretical issues which always necessitate 
to chose the correct balance between accuracy and computational cost. As such, various levels of approximation have been proposed to describe liquid water. On the one hand, a number of force fields (FF) such as TIP4P/2005, ${ }^{15}$ TIP5P, ${ }^{16}$ Thole-type (TTM) potentials, ${ }^{17-21} \mathrm{SPC} / \mathrm{E},{ }^{22}$ and others, ${ }^{4,23-26}$ have been developed to model liquid water under a wide range of temperatures and pressures. These potentials are computationally inexpensive and allow for long simulation even for large system sizes but they are limited as soon as an explicit electronic structure description is required. On the other hand, since the pioneering study by Laasonen et al. in the mid-1990's, ${ }^{27}$ density functional theory (DFT) has also been intensively used to model liquid water. Indeed, a number of DFT studies have probed the properties of water at the atomic level while rationalising the strengths and weaknesses of DFT for this particular system. ${ }^{1,28-53}$ Nowadays, it is well recognised that an accurate description of liquid water at the DFT level should combine an hybrid functional, ${ }^{35,37,43,51}$ dispersion interaction corrections ${ }^{39,41,42,44,51}$ for a proper description of the potential energy surface on the one side, and the inclusion of nuclear quantum effects (NQEs) on the other side. ${ }^{7,31,38,47,50,54}$ For instance, DiStasio et al. were able to obtain very accurate pair radial distribution functions $(g(r))$ using both an hybrid functional and dispersion interaction corrections. ${ }^{51}$ Chen and co-workers were also able to obtain a quite good structural description of liquid water using the SCAN functional. ${ }^{53}$ The inclusion of NQEs was missing in those studies but such a simulation would be computational extremely costly and hardly applicable to questions such as reactivity in aqueous solution and solvation of large molecular species.

In contrast to liquid water, liquid benzene has sustained much less interest lately, although aromatic solvents are of particular interest in the petroleum industry where benzene and its derivatives are used as solvent for asphaltenes. ${ }^{55}$ Much fewer experimental and theoretical studies were thus conducted. The structure of liquid benzene was studied by X-ray ${ }^{56}$ and neutron diffraction ${ }^{57}$ experiments and few theoretical studies were achieved using FF potentials based on a Lennard-Jones description of the intermolecular interactions. ${ }^{58-64}$

To go a step further into the description of these liquids, it is desirable to develop an 
intermediate approach in between DFT and FF which computational cost would allow for the description of large systems (of several hundreds of molecules) while conserving an accuracy close to DFT results and an explicit description of the electronic structure. The selfconsistent-charge density-functional based tight-binding approach (SCC-DFTB, also referred to second-order DFTB or DFTB2 $)^{65-68}$ meets these requirements due to the use of a minimal valence basis-set and parameterized integrals. Two important studies tested the ability of SCC-DFTB to model liquid water. In the first one, Maupin and co-workers discussed the performances of two SCC-DFTB formulations: the original formulation and a modified scheme proposed by Elstner that includes an optimized hydrogen-bond (HB) damping function. ${ }^{68,69}$ Both models lead to oxygen-oxygen, oxygen-hydrogen and hydrogen-hydrogen $g(r)$ that significantly differ from both DFT and experimental curves. In particular, they display $g_{O O}(r)$ functions that are almost flat beyond $3.5 \AA$ which is associated to an overestimated water self-diffusion coefficient. In the second one, which extends a first study published in 2011 by the same authors, ${ }^{70}$ Goyal et al. applied various formulations of third-order DFTB (referred to as DFTB3) to liquid water. ${ }^{71}$ The author showed that standard DFTB3 does not improve significantly the $g(r)$ with respect to SCC-DFTB results although an ad hoc correction of the $\mathrm{O}-\mathrm{H}$ repulsive potential, obtained by means of an iterative Boltzmann inversion procedure to tune it, could highly improve the results. These studies show that there are still possibilities for improvement of the SCC-DFTB description of liquid water, as demonstrated by another very recent study that again uses the iterative Boltzman inversion procedure to fine tune the O-H and O-O repulsive potentials. ${ }^{72}$ Although SCC-DFTB has been applied to the description of other molecular liquids, ${ }^{73}$ it has to our knowledge never been applied to liquid benzene.

The Mulliken definition of atomic charges used in the SCC-DFTB potential can be replaced by other approaches like the Class IV/Charge Model 3 (CM3) charge scheme, ${ }^{74,75}$ It can been used as a post-analysis scheme to improve the computation of electric dipoles, ${ }^{77}$ or introduced in the DFTB potential to replace the Mulliken charge. ${ }^{76}$ In the latter case, it 
appeared to improve the structural and energetic properties of polycyclic aromatic hydrocarbon $(\mathrm{PAH})$ clusters $^{76,78-80}$ and water clusters isolated, with impurities or deposited on a PAH surface. ${ }^{81-85}$ Encouraged by these results, we demonstrate in the present article how the use of an alternative formulation of the atomic charges, based on a non-symmetric repartition of the overlapping density between two centers, can improve the SCC-DFTB description of liquid water and benzene under ambient conditions. Furthermore, as mentioned above, NQEs have been shown to influence the $g(r)$ 's of liquid water within the DFT framework (see ${ }^{7}$ and references therein). Consequently, we also report the coupling of the deMonNano ${ }^{86}$ and i$\mathrm{PI}^{87}$ codes which are used to probe the impact of NQEs through the path-integral molecular dynamics (PIMD) scheme ${ }^{88-91}$ within the SCC-DFTB framework. Finally, the anisotropy of the hydration shell of a benzene molecule in water has also sustained a lot of interest lately $^{92,93}$ (and references therein) and we demonstrate that SCC-DFTB provides results of near-DFT quality for such system.

The paper is organized as follow: the computational methods and simulation details employed along the article are described in the Computational Details section. The results are presented in the Results and Discussion section where are first discussed the results obtained for liquid water, classical then quantum, and then the results for liquid benzene and a benzene molecule in liquid water. The main outcomes and perspectives are summarized in the Conclusion.

\section{Computational Details}

SCC-DFTB Potential and Weighted Mulliken Charges. Molecular dynamics simulations were performed using the SCC-DFTB formalism, ${ }^{65-68}$ an approximated DFT scheme whose computational efficiency relies on the use of an atomic minimal valence basis-set $\left\{\phi_{\mu}\right\}$ and parameterized integrals. We recently introduced a modification consisting in replacing the Mulliken atomic charges used to compute the SCC-DFTB energy by the so-called 
Weighted Mulliken charges (hereafter, called WMull) ${ }^{94,95}$ which introduce a non-symmetric bias directly into the Mulliken repartition of the overlapping density:

$$
\phi_{\mu}(r) \phi_{\nu}(r) \simeq \frac{1}{2} S_{\mu \nu}\left(\left(1+t_{\mu \nu}\right)\left|\phi_{\mu}(r)\right|^{2}+\left(1-t_{\mu \nu}\right)\left|\phi_{\nu}(r)\right|^{2}\right)
$$

where $t_{\mu \nu}$ is a free parameter ranging from -1 to 1, describing the polarity of a given chemical bond. The role of this parameter is similar to that of the empirical parameters used to compute CM3 charges, although not strictly equivalent. In the following, we investigate two approaches to determine $t_{\mu \nu}$ : (i) empirically as an atomic pair parameter $\left(t_{\mu \in A, \nu \in B}=\right.$ $t_{A B}$ ), hereafter called the Weighted Mulliken (WMull) scheme or (ii) derived from atomic orbital energies through the expression $t_{\mu \nu}=\frac{\epsilon_{\mu}}{\epsilon_{\mu}+\epsilon_{\nu}}$, hereafter called the $\epsilon$-Mulliken ( $\epsilon$ Mull) scheme. One interest of the WMull scheme with respect to the CM3 approach is its ease of implementation for periodic condition, mandatory for the present work. We may note that the WMull scheme has been recently used in an astrophysical context to describe water ice, ${ }^{94}$ to compute IR spectra of PAH clusters (unpublished results) as well as in the study of the conformational change free-energy surface of alanine dipeptide in vaccum and in liquid water. ${ }^{96}$

We also use an empirical correction term to take into account dispersion interactions ${ }^{76}$ with the atomic $C_{6}$ parameters derived by $\mathrm{Wu}$ and Yang et al.. ${ }^{97}$ We used the mio-set for Slater-Koster tables. ${ }^{66}$ All calculations were performed with the deMonNano code. ${ }^{86}$

Classical Molecular Dynamics Trajectories. The SCC-DFTB classical trajectories of liquid water were performed for 128 water molecules in a $15.64 \AA$ cubic box in the canonical ensemble at $300 \mathrm{~K}$ using a global Nose-Hoover chain of five thermostats with frequencies of $800 \mathrm{~cm}^{-1}$ and a 0.5 fs time step. ${ }^{98-100}$ In order to insure that the nature of the thermostat has no influence on the results, Figure S1 in Supporting Information compares $g(r)$ functions of liquid water obtained with the Nose-Hoover chain and the stochastic velocity rescaling thermostat with a 1.0 ps time constant. ${ }^{101}$ Comparisons with simulations performed in the microcanonical ensemble are also provided. The systems were equilibrated during 20 ps at 
$300 \mathrm{~K}$ before 100 ps of production run.

A similar procedure was conducted on two $31.28 \AA$ cubic unit cells containing 1024 water molecules each. One of these simulations was obtained using Mulliken charges, the other one with the WMull scheme with $\mathrm{t}_{\mathrm{OH}}=0.28$. These two simulations were used to characterize the voids that exist within the hydrogen bond network of the simulated liquid water. To analysis the geometry of these voids, we used the Voronoi-Delaunay method ${ }^{102}$ which is implemented in the VNP code. ${ }^{103}$ In general, two radii, namely probe $\left(R_{P}\right)$ and bottleneck $\left(R_{B}\right)$, are needed to construct the Voronoi voids. The former is used for identification of the empty space between the molecules and the latter for merging the connected regions. Here, we used $R_{P}$ and $R_{B}$ equal to 1.2 and $1.1 \AA$, respectively. The interested reader may refer to references ${ }^{104-106}$ for more details. For comparison, the voids obtained at the SCC-DFTB level were compared to those obtained using a FF simulation. In that case, a molecular dynamics simulation of TIP4P-Ew ${ }^{107}$ liquid water (4096 molecules) was performed using the GROMACS 5.0 package ${ }^{108}$ in a cubic box with an average side length $49.68 \AA$. The system was equilibrated for 2 ns with the time step of $2 \mathrm{fs}$, and the final production run was carried out over another $40 \mathrm{~ns}$ at NPT ensemble. The Nose-Hoover thermostat and the ParrinelloRahman barostat ${ }^{109}$ was used to fix the system temperature and pressure at $300 \mathrm{~K}$ and 1 bar, with coupling time constants of 2 ps and 1 ps respectively.

The SCC-DFTB simulations of liquid benzene consisted in 36 benzene molecules in a $17.47 \AA$ cubic box to respect the density of liquid benzene, $\sim 0.87$ g.cm ${ }^{-3}$, at $300 \mathrm{~K}$. In that case, a frequency of $400 \mathrm{~cm}^{-1}$ for the Nose-Hoover thermostat chain and a 0.5 fs time step were used with 100 ps of production run acquired for each simulation.

Simulations of one benzene molecule solvated in liquid water consisted in one benzene molecule and 126 water molecules in a $15.64 \AA$ cubic box, a time step of 0.5 fs, and the frequency of the thermostat was set to $800 \mathrm{~cm}^{-1}$. $200 \mathrm{ps}$ of production run were used for analysis of the simulations.

Quantum Molecular Dynamics Trajectories of Liquid Water. The SCC-DFTB 
quantum simulations were performed by combining the deMonNano code with the i-PI code developed by Ceriotti et al. ${ }^{87}$ The simulations were performed under canonical conditions using a system of 128 water molecules in a $15.64 \AA$ cubic box at $300 \mathrm{~K}$. All the simulations used the PIGLET formalism that affords to use a lower number of replicas as compared to conventional PIMD simulations. ${ }^{110,111}$ All our simulations were performed using 8 beads and a time step of 0.5 fs. In supporting information, a comparison with a PIMD simulation with 16 replicas and a PILE-g thermostat ${ }^{112}$ with a 1.0 ps time constant is presented to insure the convergence of our PIGLET results. As for the classical simulations, the systems were equilibrated during 20 ps prior to 100 ps production run.

\section{Results and Discussion}

\section{Description of Liquid Water}

Classical Description. Figure 1 provides a comparison between the $g_{O O}(r), g_{O H}(r)$ and $g_{H H}(r)$ functions obtained using different values of the $t_{\mathrm{OH}}$ parameter defined in the WMull formalism (see equation 1). We tested values going from 0.0 , which corresponds to the original Mulliken charges, to 0.58. For comparison, we report on Figure 1 the DFT curves obtained by Marsalek and Markland using the revPBE-D3 functional to benchmark the present SCC-DFTB results. ${ }^{54}$ It is well documented that different functionals lead to different $g(r)$ functions for liquid water and that few of them lead to data quantitatively comparable to the experiment. In particular, no generalized-gradient approximated (GGA) functional properly describe the structure of liquid water. As a consequence, finding reference GGADFT radial distribution functions can hardly be done and is subject to randomness. Here, we choose the revPBE-D3 calculations by Marsalek and Markland as reference as it is a GGA functional derived from PBE, that is used to generate the SCC-DFTB parameters, ${ }^{66}$ and that gives correct $g(r)$ for liquid water.

In absence of charge correction, i.e. $\mathrm{t}_{\mathrm{OH}}=0.0$, the curves we report are similar to the 

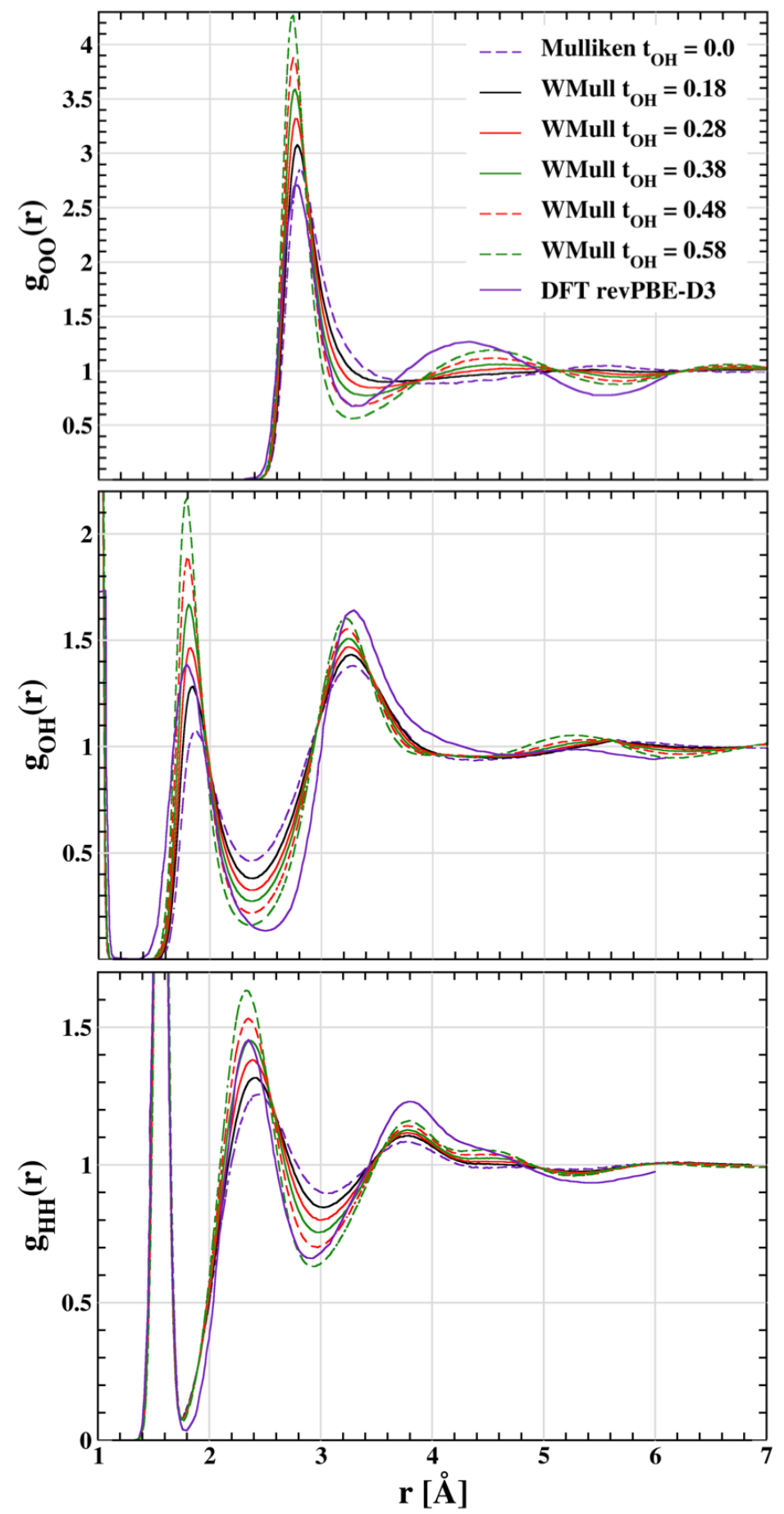

Figure 1: $g_{O O}(r)$ (top), $g_{O H}(r)$ (middle) and $g_{H H}(r)$ (bottom) functions obtained at $300 \mathrm{~K}$ for classical liquid water using different $\mathrm{t}_{\mathrm{OH}}$ parameters in the WMull formalism. Comparison with the DFT results obtained with the revPBE-D3 functional by Marsalek and Markland are provided for comparison. ${ }^{54}$ 
ones previously obtained for the description of liquid water at the SCC-DFTB level. ${ }^{69,71}$ The shortcomings of this original SCC-DFTB formulation have already been pointed out and discussed, ${ }^{69,71,113}$ we briefly remind them in the following. First, $g_{O O}(r)$ is significantly less structured than the DFT curve (see top panel of Figure 1) with a too broad first peak, the absence of minimum in the region around $3.3 \AA$ and the absence of a well defined second solvation shell. Indeed, only a very weak maximum is obtained at $\sim 5.5 \AA$ whereas the DFT curve displays such a maximum at $\sim 4.5 \AA$ as well as a minimum at $\sim 5.5 \AA$. This latter does not exist in the SCC-DFTB curve. In contrast to the $g_{O O}(r)$, the $g_{O H}(r)$ and $g_{H H}(r)$ obtained at the SCC-DFTB level display the correct shapes with the correct number of maxima and minima. Nevertheless, the $g_{O H}(r)$ in the intermolecular region (i.e. for distances larger than $1.4 \AA$, see middle panel of Figure 1) also displays a lack of structuration: (i) the $g_{O H}(r)$ value at the maximum of the first peak is too low (1.08 instead of 1.38 at the revPBE-D3 level) although its position is reasonable at $1.87 \AA$ (1.79 at the revPBE-D3 level) ; (ii) the $g_{O H}(r)$ value is too high for the first minimum and its position is shifted $(2.36 \AA$ against $2.50 \AA$ at the revPBE-D3 level) ; (iii) the second maximum is too small and slightly shifted ; (iv) there is no maximum in the region around $5.2 \AA$. Similarly, the $g_{H H}(r)$ in the intermolecular region (i.e. for distances larger than $1.8 \AA$, see bottom panel of Figure 1), presents the following differences with respect to revPBE-D3 results: (i) a too low value for the first maximum with an up-shift of $0.09 \AA$; (ii) a too high value for the first minimum with an up-shift of $0.17 \AA$; (iii) a too low value for the second maximum with a down-shift of $0.07 \AA$. All these discrepancies demonstrate that the original SCC-DFTB formulation provides a very poor description of the structure of liquid water. As demonstrated by Maupin et al. ${ }^{69}$ this is associated to very poor dynamical properties. In particular, the self-diffusion coefficient of water is $1.11 \pm 0.04 \AA^{2} \cdot \mathrm{ps}^{-1}$ at the SCC-DFTB level as compared to 0.1 and $0.23 \AA^{2} \cdot \mathrm{ps}^{-1}$ at the DFT level (using the HCTH/120 functional) ${ }^{114}$ and experimentally, ${ }^{115}$ respectively. This much too high diffusion coefficient is explained by the flatness of $g_{O O}(r)$ above $3.5 \AA$ and the absence of a well defined second solvation shell. 
When the WMull correction is introduced, the three $g(r)$ are significantly impacted. Indeed, increasing the $t_{\mathrm{OH}}$ value results in a better structuration of the $g_{O O}(r)$ which partly corrects the aforementioned defaults of the original Mulliken charges: (i) a minimum progressively appears around $3.3 \AA$; (ii) the structuration of the second solvation shell improves with the appearance of a maximum and a minimum at $\sim 4.6$ and $\sim 5.7 \AA$, respectively. If compared to the revPBE-D3 curve, the higher $t_{\mathrm{OH}}$, the better the comparison is between the SCC-DFTB and revPBE-D3 curves beyond $3 \AA$. However, as $\mathrm{t}_{\mathrm{OH}}$ increases, the height of the first peak of the $g_{O O}(r)$ also increases to very high values. The same behavior is observed for $g_{O H}(r)$ and $g_{H H}(r)$. Indeed, in both case, increasing toH makes the SCC-DFTB curves closer to the DFT ones at the first minimum and second maximum. However, a too large value leads to a too high first peak (see middle and bottom panels of Figure 1). For $g_{O H}(r)$, whatever $\mathrm{t}_{\mathrm{OH}}$, the positions of the first minimum and second maximum remain down-shifted with respect to the revPBE-D3 curve. In contrast, for $g_{H H}(r)$, increasing $\mathrm{t}_{\mathrm{OH}}$ leads to peak positions that are closer to the DFT ones.

As first conclusions, the use of improved charges clearly leads to $g(r)$ that are closer to DFT curves. In particular, it allows to recover the correct shape of the $g_{O O}(r)$ which was totally missing with the original Mulliken charges. Consequently, a much better description of the second solvation shell of the water molecules is obtained. However, from the present tests, it is clear that no $\mathrm{t}_{\mathrm{OH}}$ value can lead to perfect results. Indeed, while a better description of this second solvation shell is obtained by increasing $t_{\mathrm{OH}}$, this also leads to an over-structuration of the first solvation shell reflected in a too high first intermolecular peak in the three $g(r)$. So a balance has to be chosen. $\mathrm{t}_{\mathrm{OH}}$ values of 0.28 and 0.38 appear as good compromises to correctly describe both regions of the three $g(r)$. At this point, it is important to notice that the WMull scheme was previously used in the context of the description of water clusters. ${ }^{94}$ and in that study, the authors determined a $t_{\mathrm{OH}}$ value so as to reproduce the binding energies of water clusters previously obtained with the CM3 charge correction. Interestingly, a $\mathrm{t}_{\mathrm{OH}}$ value of 0.39 was obtained, close to the higher limit for liquid 
water determined in this work. The present results can be discussed in the light of the work by Goyal et al. on the modelling of liquid water with DFTB3. ${ }^{71}$ As for SCC-DFTB, original formulation of DFTB3 does not provide a good description of the liquid water structure. However, using a reverse Monte Carlo algorithm based on the experimental curve, which has also been done in other studies, ${ }^{116}$ the authors determined the shape of $E_{O H}^{\mathrm{rep}}$ that would leads to the a correct $g_{O O}(r)$. Interestingly, this a priori repulsive function becomes attractive in the 1.6-3.5 $\AA$ region. This demonstrates that SCC-DFTB (and DFTB3) miss some physics in the description of the water-water interaction at mid-distance and one hypothesis is the absence of atomic polarizability resulting from the use of a minimal basis set. An ad-hoc introduction of atomic polarizabilities in SCC-DFTB has already been proposed but it has not yet been considered for the description of liquid water. ${ }^{117}$

To go a step further into describing the impact of the charge correction, Figure 2 displays the $g_{O O}(r), g_{O H}(r)$ and $g_{H H}(r)$ functions obtained from the WMull scheme, with $t_{\mathrm{OH}}$ values of $0.0,0.28,0.38$, as well as from the $\epsilon$ Mull scheme. For comparison, the revPBE-D3 curves and the experimental data by Sopper and Benmore are also reported. ${ }^{118}$ In the Supporting Information, comparison with the experimental data by Skinner et al. ${ }^{119}$ for $g_{O O}(r)$ and Wikfeldt et al. for $g_{O H}(r)$ and $g_{H H}(r),{ }^{120}$ are provided and discussed. In the three cases, the $\epsilon$ Mull scheme for charge correction leads to equivalent results as the WMull scheme with $t_{\mathrm{OH}}=0.28$. The most striking feature in Figure 2 is the correct qualitative agreement between the SCC-DFTB $g(r)$ and the experimental curves. Indeed, although the match between SCC-DFTB and revPBE-D3 curves is far from being perfect due to the well-known over-structuration obtained from conventional GGA-based functionals, comparison with experiment is better. This is true for all $g(r)$ features displayed in Figure 2 except for the first peak of $g_{O O}(r)$ which is still too high even compared to experiment.

As a conclusion to this analysis, it appears that the atomic charges corrections proposed in Eq. (3) lead to a general improvement of the water $g(r)$. Within the WMull scheme, a correcting factor of $\mathrm{t}_{\mathrm{OH}}=0.28$ appears as a good compromise to recover the main features 


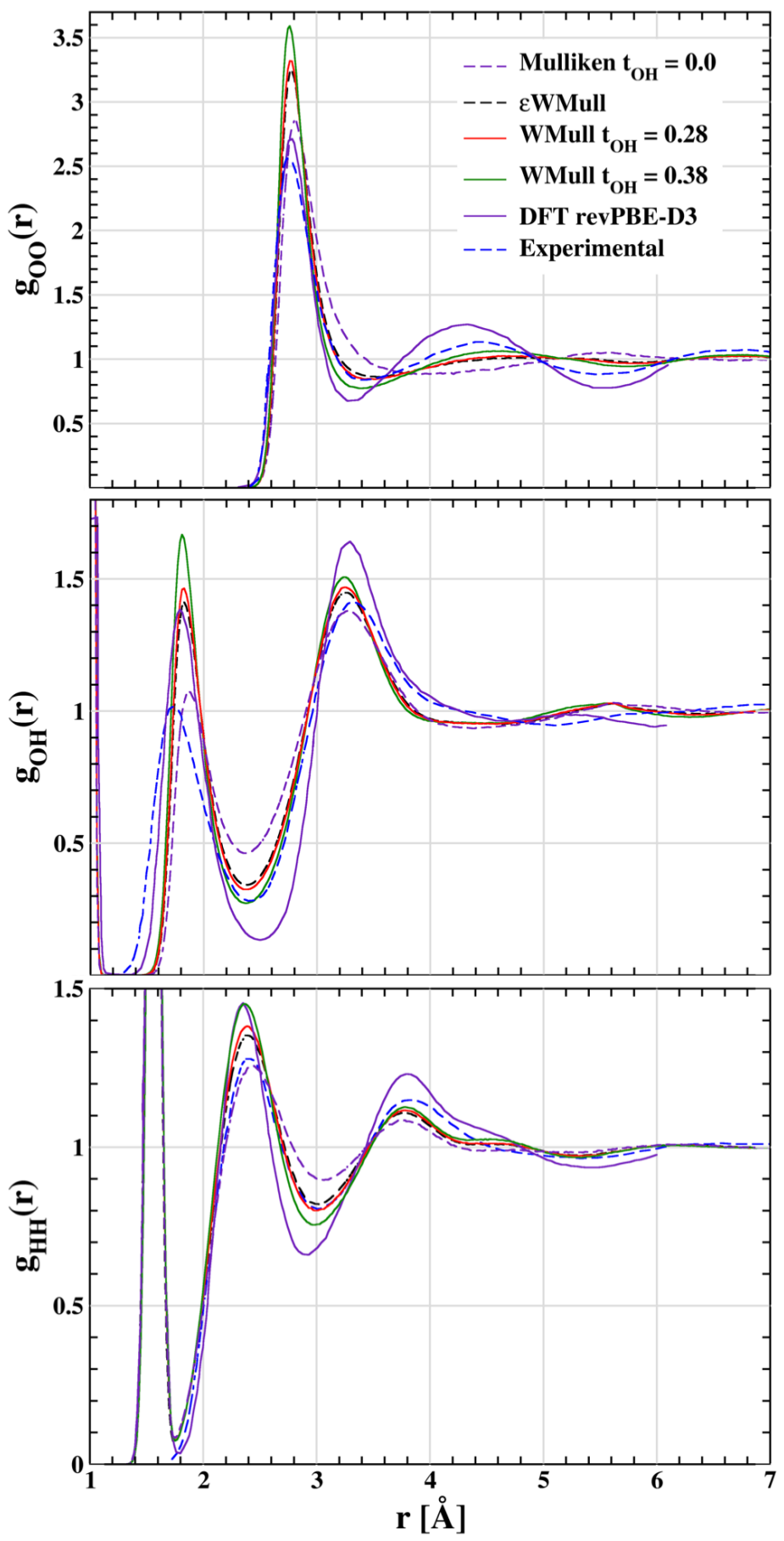

Figure 2: $g_{O O}(r)$ (top), $g_{O H}(r)$ (middle) and $g_{H H}(r)$ (bottom) obtained at $300 \mathrm{~K}$ for classical liquid water using the WMull scheme with three $\mathrm{t}_{\mathrm{OH}}$ values: $0.0,0.28$ and 0.38 , as well as the $\epsilon$ Mull scheme. The results obtained with the original Mulliken charges correspond to $\mathrm{t}_{\mathrm{OH}}=0.0$. Comparison with the DFT results obtained with the revPBE-D3 functional by Marsalek and Markland are provided for comparison, ${ }^{54}$ as well as the experimental curves of Sopper and Benmore. ${ }^{118}$ 
of $g_{O O}(r), g_{O H}(r)$ and $g_{H H}(r)$ without introducing artefacts and without additional computational cost. Interestingly, the WMull scheme with $\mathrm{t}_{\mathrm{OH}}=0.28$ gives similar results as the $\epsilon$ Mull scheme, therefore also suitable. This gain in accuracy is also true for other properties. In particular, the self-diffusion coefficient of water, which is $1.11 \pm 0.04 \AA^{2} \cdot \mathrm{ps}^{-1}$ at the original SCC-DFTB level, ${ }^{69}$ is $0.78 \AA^{2} \cdot \mathrm{ps}^{-1}$ using $\mathrm{t}_{\mathrm{OH}}=0.28$. This lower value results from the more structured liquid water obtained with the improved charges. The heat of vaporization was also calculated following the procedure described in ref. ${ }^{69}$ Using $_{\mathrm{OH}}=0.28$ and 0.38 leads to values of 7.93 and $9.17 \mathrm{kcal} . \mathrm{mol}^{-1}$, respectively, which are both closer to the experimental value, $10.50 \mathrm{kcal} . \mathrm{mol}^{-1}$, than the original SCC-DFTB formulation which gives $4.09 \mathrm{kcal} \cdot \mathrm{mol}^{-1}$. This arises from the better description of the hydrogen bonding energy when improved charges are included. Finally, Figure S5 of the Supporting Information presents how the proton-transfer energy barrier changes with the inclusion of the weighted Mulliken charges. As can be seen, while the original SCC-DFTB formulation is characterized by a very low energy barrier, both $\mathrm{t}_{\mathrm{OH}}=0.28$ and 0.38 lead to a drastic improvement as compared too MP2/aug-cc-pVTZ calculations, although still underestimating the barrier. Although more complete investigations are necessary, these preliminary results are encouraging in the view of describing reactive processes in liquid water using SCC-DFTB with improved charges.

As mentioned above, an optimized $t_{\mathrm{OH}}$ value of 0.39 was obtained for water clusters based on the energetics of finite-size systems. ${ }^{94}$ This demonstrates that, although an overall improvement of the description of liquid water can be obtained using the WMull and $\epsilon$ Mull schemes, some empirical insight must be introduced for a refined tuning of the charge correction. Finally, it is worth mentioning that such correction does not solve all the discrepancies of DFTB for the simulations of liquid water. In particular, the appearance of large voids in the simulations, which was already highlighted by Choi et al., ${ }^{113}$ is still present as demonstrated in Figure 3. Indeed, the distributions of void volumes at the SCC-DFTB level is found to be very similar to the TIP4P-Ew distribution except for void volumes larger than $200 \AA^{3}$ for which they are higher. Unfortunately, the WMull correction is found to 
have nearly no effect. This was expected as Choi et al. attributed this deficiency to the too intense first peak in $g_{O O}(r)$. This suggests that further improvement of the $\mathrm{O}-\mathrm{H}$ and $\mathrm{O}-\mathrm{O}$ SCC-DFTB parametrization will be needed to attenuate the intensity of the first $g_{O O}(r)$ peak in order to counterpart this default.

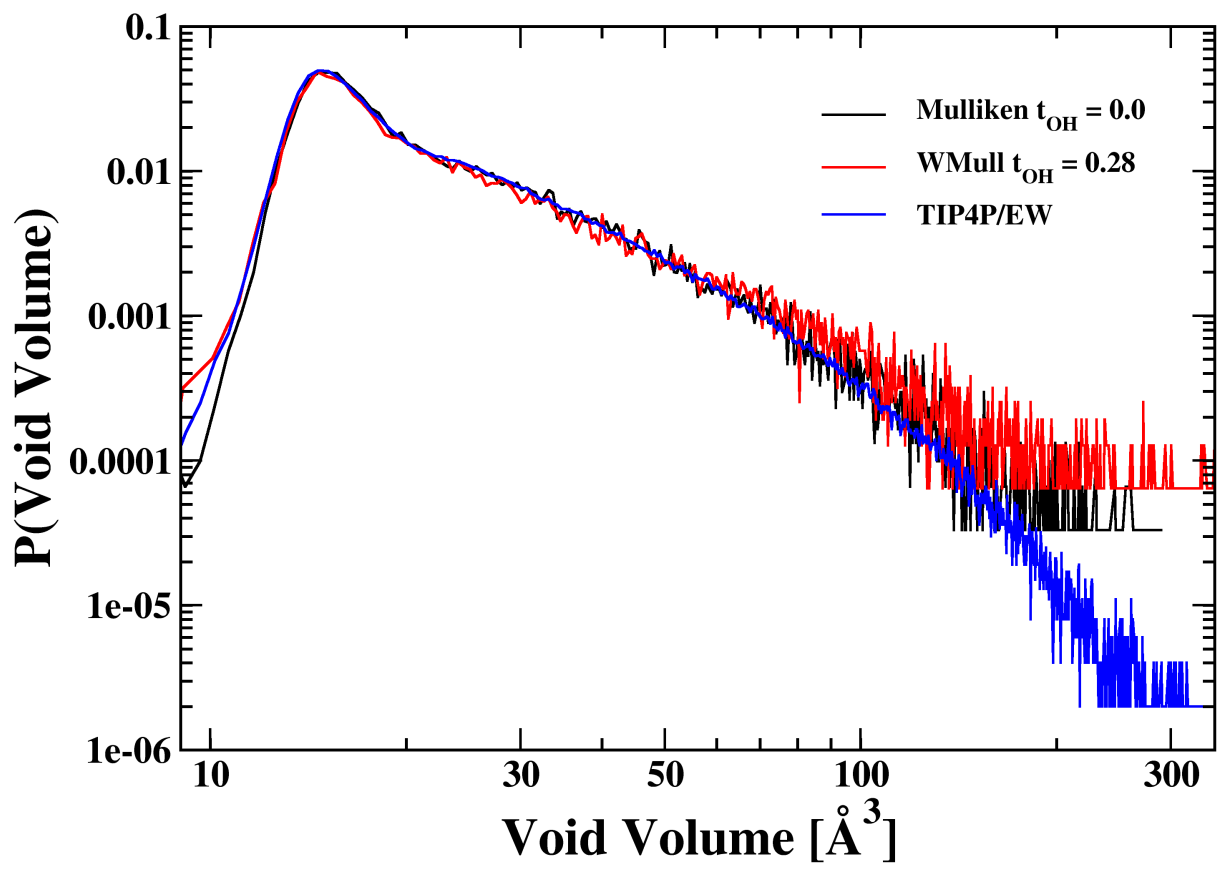

Figure 3: Volume distribution of voids in simulations of 1024 water molecules using Mulliken (black curve) and Wmull with $\mathrm{t}_{\mathrm{OH}}=0.28$ (red curve) of liquid water at $\mathrm{T}=300 \mathrm{~K}$. For comparison, the volume distribution of voids obtained from a TIP4P $/ \mathrm{Ew}^{107}$ simulation of 4096 water molecules is also presented (blue curve).

Impact of NQEs. As stated in introduction, the role of nuclear quantum effect can be non negligeable on the liquid water $g(r)$, in particular $g_{O H}(r)$ and $g_{H H}(r) .7,31,38,47,50,54$ To probe their impact on the present simulations, we now present simulations performed using the PIMD technique as implemented in the i-PI code. ${ }^{87}$ Figure 4 provides a comparison between the $g_{\mathrm{OO}}(r), g_{\mathrm{OH}}(r)$ and $g_{H H}(r)$ functions obtained at $300 \mathrm{~K}$ using the WMull scheme with $t_{\mathrm{OH}}=0.28$ and 0.58 . Results from both classical and quantum simulations are displayed along with the experimental data of Sopper and Benmore. ${ }^{118}$

The $g_{O O}(r)$ is only slightly affected by NQEs, the main difference with classical simulation being a small decrease of the first peak intensity. For $\mathrm{t}_{\mathrm{OH}}=0.28$, behind $3 \AA$ the classical 
and quantum curves are identical. For $\mathrm{t}_{\mathrm{OH}}=0.58$, slight differences exist between the classical and quantum curves as the first and second maximum are slightly higher and lower, respectively, in the quantum simulation. Those effects on $g_{O O}(r)$ are expected from PIMD simulations and have already been highlighted theoretically. ${ }^{38,54}$ This is also what is observed experimentally when going from $\mathrm{H}_{2} \mathrm{O}$ to $\mathrm{D}_{2} \mathrm{O} .{ }^{118}$ Interestingly, Figure 4 demonstrates that the impact of NQEs is stronger when applied to a more structured liquid water, in the present case going from $\mathrm{t}_{\mathrm{OH}}=0.28$ to 0.58 . This explains why in the $\mathrm{SCC}-\mathrm{DFTB} g_{O O}(r)$ case the impact of NQEs is rather low while it is much stronger when considering DFT simulations that lead to a more structured liquid. ${ }^{31,38,50,52,54}$

As expected, the impact of NQEs is stronger on $g_{O H}(r)$ and $g_{H H}(r)$. As for $g_{O O}(r)$, the effect is enhanced for $\mathrm{t}_{\mathrm{OH}}=0.58$ with resepct to 0.28 , as the liquid is more structured and thus more sensitive to NQEs. More specifically, the two maxima of $g_{O H}(r)$ at 1.82 and $3.25 \AA$ decrease while the minimum at $2.38 \AA$ increases. Overall, inclusion of NQEs leads to a better agreement with the experimental curve although the aforementioned discrepancies remain. These effects are similar to those reported from DFT simulations. ${ }^{38,50,54}$ Although the same general remarks can be drawn for $g_{H H}(r)$, the inclusion of NQEs now makes the $\mathrm{t}_{\mathrm{OH}}=0.28$ curve match very well with the experimental data up to $\sim 4 \AA$.

To conclude, combining the PIMD algorithm to the present SCC-DFTB potential leads to $g(r)$ modifications similar to what was reported at the DFT level. NQEs leads to $g(r)$ functions that are closer to the experimental data in terms of intensities but do not correct the fundamental errors of the SCC-DFTB potential. The present simulations also show that their impact on the intensities varies with charge correction.

\section{Description of Liquid Benzene}

In this section, we focus on the description of liquid benzene restricting the study to classical MD. We investigate the influence of the $t_{\mathrm{CH}}$ value on the carbon-carbon $\left(g_{C C}(r)\right)$, center of mass-center of mass $\left(g_{C M-C M}(r)\right)$ and all atoms-all atoms $\left(g_{B z B z}(r)\right)$ intermolecular pair 

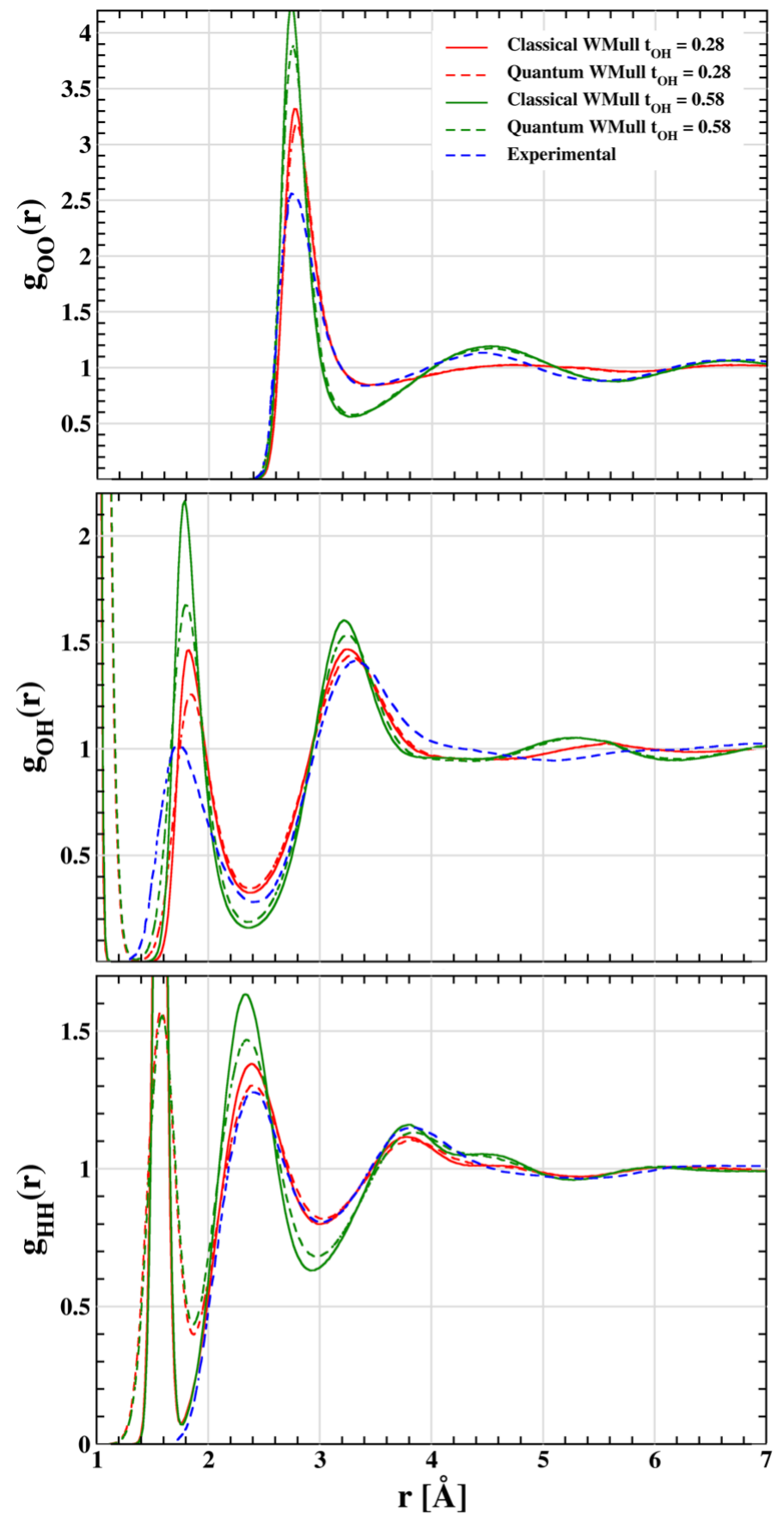

Figure 4: $g_{O O}(r)$ (top), $g_{O H}(r)$ (middle) and $g_{H H}(r)$ (bottom) obtained at $300 \mathrm{~K}$ for classical and quantum liquid water using the WMull scheme with two $\mathrm{t}_{\mathrm{OH}}$ values: 0.28 and 0.58 . The experimental curves of Sopper and Benmore are provided for comparison. ${ }^{118}$ 
radial distribution functions. Three $t_{\mathrm{CH}}$ values were tested: 0 , corresponding to the Mulliken scheme, 0.30 which was used to study PAHs in interaction with water ice, ${ }^{94}$ and the intermediate value of 0.15 . The results are reported in Figure 5 along with experimental ${ }^{56}$ and FF results from other works. ${ }^{59,62}$ The limitation of our approach in comparison with previous studies is the size of the system: we are only able to compare our data for C-C and $\mathrm{Bz}-\mathrm{Bz}$ distances up to $8 \AA$ although experimental and other $\mathrm{FF}$ data are provided for distances up to about $15 \AA$.

As can be seen in Figure 5, the influence of WMull is little for $g_{C C}(r)$, but can be clearly seen for $g_{C M-C M}(r)$, and also for $g_{B z B z}(r)$ to a lesser extent, as an increase of the $t_{C H}$ value leads to enhanced structuration, similarly to what we observe for water. Regarding $g_{C C}(r)$, one shoulder is found at $4 \AA$ (intensity of 0.9 ), and two maxima are found at $4.9 \AA$ (1.08) and $6 \AA$ (1.16). The SCC-DFTB $g_{C C}(r)$ appears in very good agreement with FF results obtained with an intermolecular potential aiming at describing anisotropic interactions (Anisotropic United Atoms intermolecular potential) ${ }^{121}$ and to a lesser extent with the experimental ones. ${ }^{56,58}$ Indeed, similarly to FF simulations, the SCC-DFTB approach appears to underestimate the correlation fluctuations of the $\mathrm{CC}$ pairs for all $\mathrm{t}_{\mathrm{CH}}$ values.

Regarding $g_{C M-C M}(r)$, one broad peak is observed for distances smaller than $8 \AA$, with a maximum located at $5.4 \AA$ in the case of Mulliken charges, down to $5.2 \AA$ for a $\mathrm{t}_{\mathrm{CH}}$ value of 0.30 . When increasing $t_{\mathrm{CH}}$ the peak becomes more assymetric, with a maximum slightly shifted towards smaller $r$. This can be explained as follow: based on the results for the benzene dimer in the gas phase, we show that the maximum at 5.2-5.4 $\AA$ is due to T-shape configurations between benzene molecules, the equilibrium intermolecular distance for such configuration in the gas phase being $\sim 4.8 \AA .{ }^{76}$ As an increase of $t_{\mathrm{CH}}$ leads to an enhanced $\mathrm{C}-\mathrm{H}$ bond polarity, the CM-CM distance is expected to decrease when increasing $\mathrm{t}_{\mathrm{CH}}$. Interestingly, the starting rise of $g_{C M-C M}(r)$ starts at shorter distance, which would be more in line with the intermolecular distance within benzene molecules in $\pi$ interaction $(3.5 \AA$ in the gas phase $\left.{ }^{76}\right)$. Below $4.0 \AA, g_{C M-C M}(r)$ diminishes as $t_{\mathrm{CH}}$ increases. This can be understood 

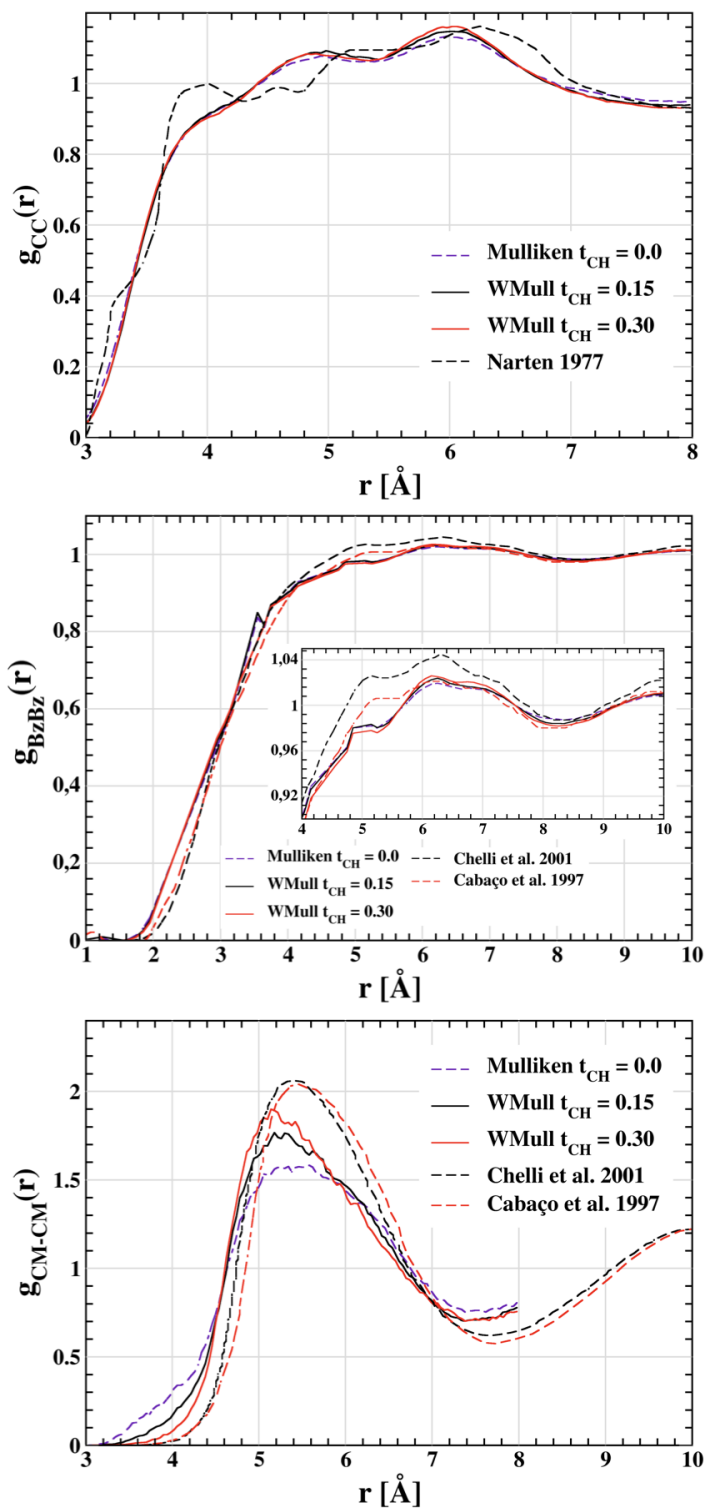

Figure 5: Influence of the WMull parameter on the $g_{C C}(r)$ (top), $g_{B z B z}(r)$ (middle) and $g_{C M-C M}(r)$ (bottom) functions obtained at $300 \mathrm{~K}$ for a classical 36 benzene molecules simulation. The results obtained with the original Mulliken charges $\left(\mathrm{t}_{\mathrm{CH}}=0.0\right)$ are provided along with two values of $t_{\mathrm{CH}}, 0.15$ and 0.30 . For $g_{B z B z}(r)$ and $g_{C M-C M}(r)$, comparison with previous theoretical results performed with FF are provided for comparison. ${ }^{59,62}$ For $g_{C C}(r)$, the experimental curve of Narten is reported. ${ }^{56}$ The inset of the middle panel is a zoom in the 4-10 A region. 
because, as mentioned above, the WMull scheme increases the C-H bond polarity and therefore is expected to lead to a higher T-shape $/ \pi$ stacking ratio. These curves, not accessible by experiments, can be compared to those obtained from FF simulations. ${ }^{59,62}$ Increasing $\mathrm{t}_{\mathrm{CH}}$ improves the agreement with the FF results as it tends to increase the intensity of the peak maximum. However it remains more asymmetric. This is expected as the anisotropic character of the interactions is inherently described with SCC-DFTB whereas it is not the case for most FF potentials. Finally, for $g_{B z B z}(r)$, the agreement is very good with the FF curve obtained by Cabacao et al. ${ }^{59}$ with the main discrepancy located in the 4.9-5.1 $\AA$ region where the SCC-DFTB intensity is lower. The SCC-DFTB $g_{B z B z}(r)$ curve is also found slightly lower than the FF curve published by Chelli et al.. ${ }^{62}$ To conclude, it appears that the influence of the WMull scheme is only visible on $g_{C M-C M}(r)$ as increasing the $t_{\mathrm{CH}}$ value slightly favor T-shape configurations while decreasing CM-CM distance for this configuration. As the best agreement with previous $\mathrm{FF}$ simulations is obtained for $t_{\mathrm{CH}}=0.30$, this value can be considered for further studies. However, the agreement with experimental data is not perfect as the structuration of $g_{C C}(r)$ remains underestimated at the SCC-DFTB level. From a dynamical point of view, $\mathrm{t}_{\mathrm{CH}}=0.30$ leads to a self-diffusion coefficient of benzene of $1.33 \mathrm{~cm}^{2} \cdot \mathrm{s}^{-1}$ as compared to $2.27 \mathrm{~cm}^{2} \cdot \mathrm{s}^{-1}$ at $298 \mathrm{~K}$ experimentally. ${ }^{122}$ Although the SCCDFTB value is half the experimental one, it is similar to the one determined by Cabaço et al., $1.2 \mathrm{~cm}^{2} . \mathrm{s}^{-1}{ }^{59}$ This demonstrates that, similarly to liquid water, there is still room for improvement of the SCC-DFTB model although the present agreement with experimental data is reasonable.

\section{Application: Benzene Molecule Solvated by Water}

In the view to describe the behaviour of asphaltene molecules and clusters at a aromatic solvent/water interface, one has to first evaluate the accuracy of SCC-DFTB to describe the interaction of aromatic hydrocarbons with liquid water. We therefore conducted SCC-DFTB simulations of one benzene molecule solvated in water using $t_{\mathrm{OH}}, t_{\mathrm{CH}}$ and $t_{\mathrm{CO}}$ values of 0.28 , 
0.30 and 0.08 , respectively. The first two values are determined in the present work, the third one is taken from reference. ${ }^{94}$

We calculated the radial distribution functions between the center of mass of benzene and the oxygen atoms of water molecules $\left(g_{C M-O}(r)\right)$ located in a cone of angle $\theta_{\text {axial }}$ around the benzene axial axis or $\theta_{\text {equatorial }}$ from the equatorial plane (see inset in Figure 6), as previously achieved by Choudary et al. ${ }^{93}$ from DFT and DFT-D simulations, DFT refering to BLYP and DFT-D to BLYP plus Grimme dispersion. When comparing the SCC-DFTB $g(r)$ reported in Figure 6 (a) and (b) with those reported in Figure 2 (a) and (c) of ref., ${ }^{93}$ respectively, the agreement between the SCC-DFTB and the DFT-D results is found to be extremely good. The dependence of $g_{C M-O}(r)$ on the cone angle illustrates the anisotropy of the solvation shell of a benzene solute within liquid water, as studied in details by Choudary et al.. ${ }^{93}$ Next two paragraphs are dedicated to a more detailed description of the SCC-DFTB results and to their comparison with $\operatorname{DFT}(-\mathrm{D})$ simulations.

Focusing on the evolution of $g_{C M-O}(r)$ as a function of $\theta_{\text {axial }}$ (Figure $6(\mathrm{a})$ ), a very sharp peak is observed at $\mathrm{r}=3.1 \AA(\mathrm{g}=6.3)$ for an angle of $15^{\circ}$. In terms of intermolecular orientation, this corresponds to the water molecule interacting via a hydrogen atom with the $\pi$ cloud of benzene, that we previously found as the most stable configuration of the isolated benzene-water dimer within our SCC-DFTB approach. ${ }^{81,123}$ The $r=3.1 \AA$ value is the smallest possible distance that can be obtained for such dimer. When increasing $\theta_{\text {axial }}$, the sharpness of the peak decreases, it becomes flatter up to $\theta=45^{\circ}$ and becomes a shoulder for $\theta_{\text {axial }}>45^{\circ}$. This is due to the progressive average of water-benzene orientations with $\theta_{\text {axial }}$. The agreement for both peak position and intensity between the SCC-DFTB and the DFT-D results is very satisfactory. For $\theta_{\text {axial }}=15^{\circ}$, we obtain a peak position of $3.1 \AA(\mathrm{g}=6.3)$ whereas a value of $3.2 \AA$ ( $\mathrm{g}=6.8$ ) was obtained at the DFT-D level (see Figure 3 (a) of ref. ${ }^{93}$ for comparison). The evolution of the peak as a function of $\theta_{\text {axial }}$ is identical (see Figure 2 (a) of ref. ${ }^{93}$ for comparison). Interestingly, the agreement of SCC-DFTB with DFT-D results is better (although not perfect) than with DFT without dispersion. Considering larger CM-O 
distances for $\theta=15^{\circ}$, the first "axial" minimum in the $g_{C M-O}(r)$ curve is found at a shorter distance with SCC-DFTB than with DFT (4.2 against $\sim 4.8 \AA$ ) but with a similar intensity $(\sim 0.5)$. As this minimum corresponds to the benzene interacting with one water molecule through its oxygen atom, it illustrates the difference between the oxygen-carbon potential at the SCC-DFTB and DFT-D levels. At the DFT level, the distance is even longer (see Figure 3 (a) of ref. ${ }^{93}$ ). The peak corresponding to the second solvation shell appears broader and less structured at the SCC-DFTB level, starting at shorter CM-O distance ( 4.3 vs $\sim 5.0 \AA$ at the DFT-D level).

Focusing on the evolution of $g_{C M-O}(r)$ as a function of $\theta_{\text {equatorial }}$ (Figure $6(\mathrm{~b})$ ), a sharp peak is observed at $\mathrm{r}=4.7 \AA(\mathrm{g}=2.2)$ for $\theta_{\text {equatorial }}=15^{\circ}$. When $\theta_{\text {equatorial }}$ increases, the position of this peak is slightly shifted to smaller $r$ down to $r=4.6 \AA$ with an intensity decrease down to $\mathrm{g}=1.6$ for $\theta_{\text {equatorial }}=75^{\circ}$. The $\mathrm{CM}-\mathrm{O}$ distance corresponds to the benzene-water dimer conformation in which the water molecule interacts through its oxygen atom with the $\mathrm{H}$ atoms of benzene in its plane. We found that such conformation was slightly less stable at the SCC-DFTB level than the $\pi$-H conformation or the dimers in the gas phase. ${ }^{123}$ Besides, when $\theta_{\text {equatorial }}$ increases, a shoulder appears for $r$ values between 3 and $4 \AA$, which corresponds to the $\pi$-H intermolecular interactions accounting for the sharp peak observed at $\theta_{\text {axial }}=15^{\circ}$ (see above). The minimum obtained at $\sim 6.4 \AA$ is steady in position and intensity $(\mathrm{g}=0.8)$ when $\theta_{\text {equatorial }}$ increases, and similar steadiness is found for the next maximum located at $\mathrm{r}=7.7 \AA(\mathrm{g}=1.2)$. These behaviors are found to be identical to those determined at the DFT-D level (see Figure 3 (c) of ref. ${ }^{93}$ ).

Such a good agreement with one of the most accurate simulation that can be achieved on that type of system validates the description of aromatic molecule-liquid water intermolecular interactions within our SCC-DFTB scheme and, due to its computational efficiency, opens the way to further applications dedicated to the study of aromatic solvent/water interfaces. 

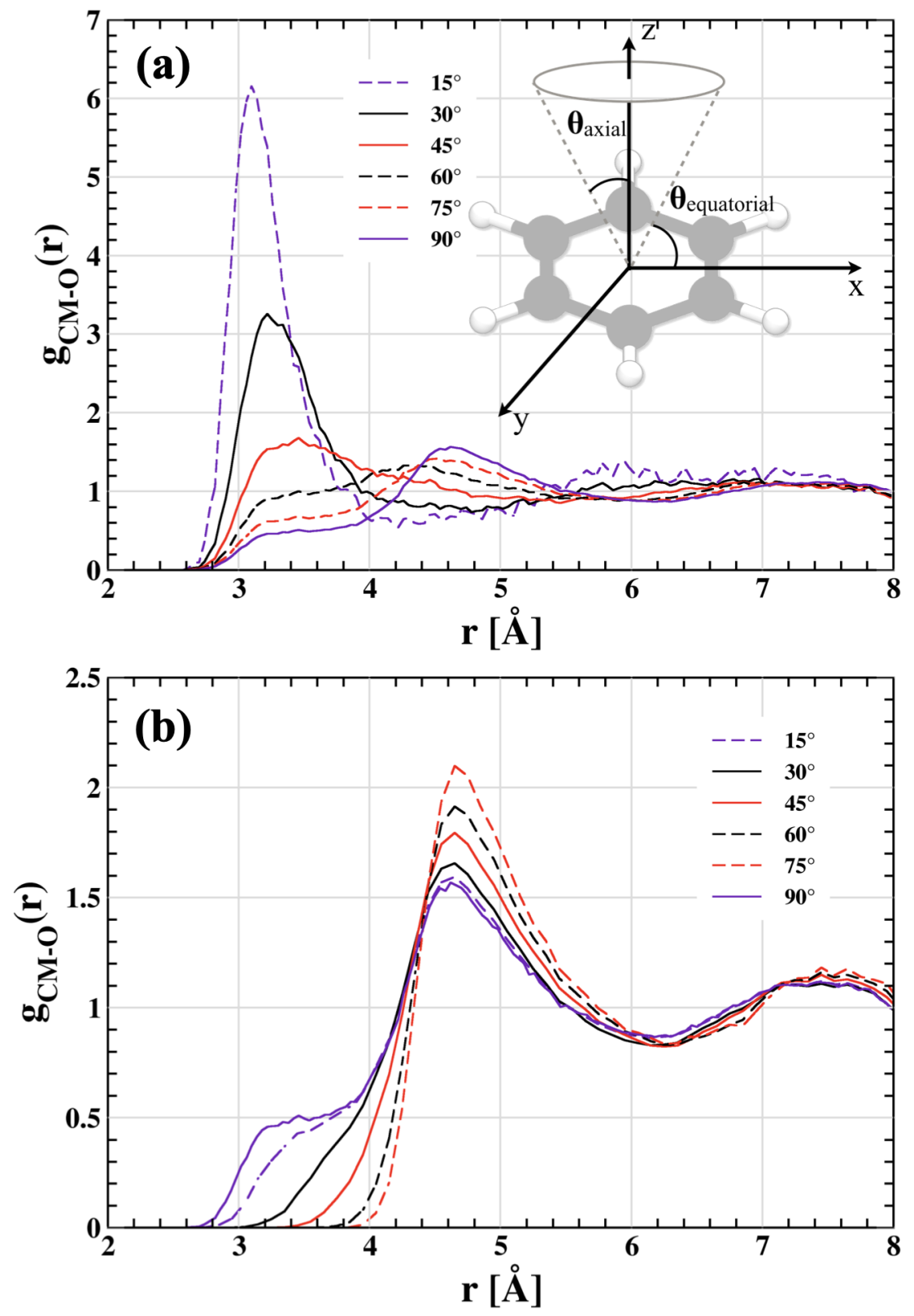

Figure 6: $g_{C M-O}(r)$ obtained for benzene solvated by water at $300 \mathrm{~K}$ (classical MD) using the WMull scheme with $\mathrm{t}_{\mathrm{OH}}=0.28, \mathrm{t}_{\mathrm{CH}}=0.30$ and $\mathrm{t}_{\mathrm{CO}}=0.08$. The curves were obtained for fixed $\theta_{\text {axial }}$ (a) and $\theta_{\text {equatorial }}(\mathrm{b})$ (see scheme for the definition of $\theta_{\text {axial }}$ and $\theta_{\text {equatorial }}$ angles). These curves can be compared to those of the top of Figure 2 in Choudary et al. ${ }^{93}$ 


\section{Conclusions}

In this work, we benchmarked the recent introduction of Weighted Mulliken charges in the SCC-DFTB potential for the description of liquids. Those charges allow for a better description of bond polarities as compared to the original Mulliken charges. Three systems were considered: liquid water, liquid benzene, and a benzene molecule in water. In the case of liquid water, the main effect of the improved charges is to increase the structuration of the $g(r)$. These lead to a significant improvement of the three pair radial distribution functions of liquid water with respect to those obtained with the original Mulliken charges. The improvements are obtained at no computational cost and no re-parametrization of the SCC-DFTB integrals is required. This study is thus a step forward in the description of liquid water at the SCC-DFTB level that demonstrates the need for using well defined atomic charges. However, all the actual limitations of the SCC-DFTB formalism cannot be solved this way, and an accurate description of liquid water at this level of theory will require further developments. We also tested the impact of NQEs on the $g(r)$ using the PIMD formalism. The effects are similar to those observed at the DFT level. They mainly consist in a slight decrease of the structuration of the $g_{O H}(r)$ and $g_{H H}(r)$ curves as compared so classical simulations.

Correction of the atomic charges also leads to a good description of the structure of aromatic hydrocarbon solvents, although the effect of the charge definition is more limited than for liquid water. More importantly, we can achieve a rather good description of a benzene molecule in liquid water. This, in addition to studies performed on aqueous molecular clusters in the gas phase and alanine dipeptide in water, ${ }^{85,95,96}$ suggests that the actual formulation of the SCC-DFTB potential, although not perfect, can be used to describe the solvation of molecules in liquid water. In that domain the efficiency of the SCC-DFTB formalism could be of use to circumvent the computational cost of DFT simulations. Of course, further investigations would be needed to check the accuracy of the presently proposed charges for a larger variety of molecules. However, our results suggest that benzene/toluene solvent at 
the interface with water could be studied at this level of theory.

\section{Supporting Information Available}

Discussion on the impact of the thermostat on the pair radial distribution functions of liquid water. Discussion on the convergence of the PIMD Simulations. Comparison of the theoretical $g(r)$ to additional experimental data are provided as well as the SCC-DFTB proton-transfer energy barrier as compared to MP2/aug-cc-pVTZ calculations. This material is available free of charge via the Internet at http://pubs.acs.org/.

\section{Acknowledgement}

The author thank the supercomputing facility of Toulouse III University, CALMIP, for allocation of computer resources (Project P1320 and P17002). We also would like to thank Elise Duboué-Dijon for fruitful discussions. This work has been funded by the Agence Nationale de la Recherche (ANR) project MUSCOFI ANR-18-CE05-0028.

\section{References}

(1) Gillan, M. J.; Alfè, D.; Michaelides, A. Perspective: How Good is DFT for Water? J. Chem. Phys. 2016, 144, 130901.

(2) Pettersson, L. G. M.; Henchman, R. H.; Nilsson, A. Water-The Most Anomalous Liquids. Chem. Rev. 2016, 116, 7459-7462.

(3) Gallo, P.; Amann-Winkel, K.; Angell, C. A.; Anisimov, M. A.; Caupin, F.; Chakravarty, C.; Lascaris, E.; Loerting, T.; Panagiotopoulos, A. Z.; Russo, J. et al. Water: A Tale of Two Liquids. Chem. Rev. 2016, 116, 7463-7500. 
(4) Cisneros, G. A.; Wikfeldt, K. T.; Ojamäe, L.; Lu, J.; Xu, Y.; Torabifard, H.; Bartók, A. P.; Csányi, G.; Molinero, V.; Paesani, F. Modeling Molecular Interactions in Water: From Pairwise to Many-Body Potential Energy Functions. Chem. Rev. 2016, 116, 7501-7528.

(5) Fransson, T.; Harada, Y.; Kosugi, N.; Besley, N. A.; Winter, B.; Rehr, J. J.; Pettersson, L. G. M.; Nilsson, A. X-Ray and Electron Spectroscopy of Water. Chem. Rev. 2016, 116, 7551-7569.

(6) Amann-Winkel, K.; Bellissent-Funel, M.-C.; Bove, L. E.; Loerting, T.; Nilsson, A.; Paciaroni, A.; Schlesinger, D.; Skinner, L. X-Ray and Neutron Scattering of Water. Chem. Rev. 2016, 116, 7570-7589.

(7) Ceriotti, M.; Fang, W.; Kusalik, P. G.; McKenzie, R. H.; Michaelides, A.; Morales, M. A.; Markland, T. E. Nuclear Quantum Effects in Water and Aqueous Systems: Experiment, Theory, and Current Challenges. Chem. Rev. 2016, 116, 75297550 .

(8) Cho, C. H.; Singh, S.; Robinson, G. W. Understanding all of Water's Anomalies with a Nonlocal Potentials. J. Chem. Phys. 1997, 10\%, 7979-7988.

(9) Robinson, G. W.; Cho, C. H.; Urquidi, J. Isosbestic Points in Liquid Water: Further Strong Evidence for the Two-State Mixture Model. J. Chem. Phys. 1999, 111, 698702.

(10) Stanley, H. E.; Buldyrev, S. V.; Canpolat, M.; Mishima, O.; Sadr-Lahijany, M. R.; Scala, A.; Starr, F. W. The Puzzling Behavior of Water at Very Low Temperature. Invited Lecture. Phys. Chem. Chem. Phys. 2000, 2, 1551-1558.

(11) Errington, J. R.; Debenedetti, P. G. Relationship between Structural Order and the Anomalies of Liquid Water. Nature 2001, 409, 318-321. 
(12) Angell, C. A. Insights into Phases of Liquid Water from Study of Its Unusual GlassForming Properties. Science 2008, 319, 582-587.

(13) Pallares, G.; El Mekki Azouzi, M.; Gonzalez, M. A.; Aragones, J. L.; Abascal, J. L. F.; Valeriani, C.; Caupin, F. Anomalies in Bulk Supercooled Water at Negative Pressure. Proc. Natl. Acad. Sci. U. S. A. 2014, 111, 7936-7941.

(14) Perakis, F.; Marco, L. D.; Shalit, A.; Tang, F.; Kann, Z. R.; Kühne, T. D.; Torre, R.; Bonn, M.; Nagata, Y. Vibrational Spectroscopy and Dynamics of Water. Chem. Rev. 2016, 116, 7590-7607.

(15) Abascal, J. L. F.; Vega, C. A General Purpose Model for the Condensed Phases of Water: TIP4P/2005. J. Chem. Phys. 2005, 123, 234505.

(16) Mahoney, M. W.; Jorgensen, W. L. A Five-Site Model for Liquid Water and the Reproduction of the Density Anomaly by Rigid, Nonpolarizable Potential Functions. J. Chem. Phys. 2000, 112, 8910-8922.

(17) Thole, B. Molecular Polarizabilities Calculated with a Modified Dipole Interaction. Chem. Phys. 1981, 59, 341-350.

(18) Burnham, C.; Li, J.; Xantheas, S.; Leslie, M. The Parametrization of a Thole-Type All-Atom Polarizable Water Model from First Principles and its Application to the Study of Water Clusters $(\mathrm{n}=2-21)$ and the Phonon Spectrum of Ice Ih. J. Chem. Phys. 1999, 110, 4566-4581.

(19) Burnham, C.; Xantheas, S. S. Development of Transferable Interaction Models for Water. IV. A Flexible, All-Atom Polarizable Potential (TTM2-F) Based on Geometry Dependent Charges Derived from an Ab Initio Monomer Dipole Moment Surface. J. Chem. Phys. 2002, 116, 5115. 
(20) Burnham, C.; Xantheas, S. S. Development of Transferable Interaction Models for Water. III. Reparametrization of an All-Atom Polarizable Rigid Model (TTM2-R) from First Principles. J. Chem. Phys. 2002, 116, 1500.

(21) Fanourgakis, G. S.; Xantheas, S. S. The Flexible, Polarizable, Thole-Type Interaction Potential for Water (TTM2-F) Revisited. J. Phys. Chem. A 2006, 110, 4100-4106.

(22) Berendsen, H. J. C.; Grigera, J. R.; Straatsma, T. P. The Missing Term in Effective Pair Potentials. J. Phys. Chem. 1987, 91, 6269-6271.

(23) Jorgensen, W. L.; Chandrasekhar, J.; Madura, J. D.; Impey, R. W.; Klein, M. L. Comparison of Simple Potential Functions for Simulating Liquid Water. J. Chem. Phys. 1983, 79, 926-935.

(24) Habershon, S.; Markland, T. E.; Manolopoulos, D. E. Competing Quantum Effects in the Dynamics of a Flexible Water Model. J. Chem. Phys. 2009, 131, 024501-11.

(25) Wu, Y.; Tepper, H. L.; Voth, G. A. Flexible Simple Point-Charge Water Model with Improved Liquid-State Properties. J. Chem. Phys. 2006, 124, 024503.

(26) Paesani, F.; Zhang, W.; Case, D. A.; Cheatham, T. E.; III,; Voth, G. A. An Accurate and Simple Quantum Model for Liquid Water. J. Chem. Phys. 2006, 125, 184507-11.

(27) Laasonen, K.; Sprik, M.; Parrinello, M.; Car, R. "Ab initio" Liquid Water. J. Chem. Phys. 1993, 99, 9080-9089.

(28) Sprik, M.; Hutter, J.; Parrinello, M. Ab initio Molecular Dynamics Simulation of Liquid Water: Comparison of Three Gradient-Corrected Density Functionals. J. Chem. Phys. 1996, 105, 1142-1152.

(29) Silvestrelli, P. L.; Parrinello, M. Water Molecule Dipole in the Gas and in the Liquid Phase. Phys. Rev. Lett. 1999, 82, 3308-3311. 
(30) Grossman, J. C.; Schwegler, E.; Draeger, E. W.; Gygi, F.; Galli, G. Towards an Assessment of the Accuracy of Density Functional Theory for First Principles Simulations of Water. J. Chem. Phys 2004, 120, 300-311.

(31) Chen, B.; Ivanov, I.; Klein, M. L.; Parrinello, M. Hydrogen Bonding in Water. Phys. Rev. Lett. 2003, 91, 215503.

(32) Ramirez, R.; Lopez-Ciudad, T.; P, P. K.; Marx, D. Quantum Corrections to Classical Time-Correlation Functions: Hydrogen Bonding and Anharmonic Floppy Modes. J. Chem. Phys. 2004, 121, 3973-3983.

(33) Kuo, I.-F. W.; Mundy, C. J.; McGrath, M. J.; Siepmann, J. I.; VandeVondele, J.; Sprik, M.; Hutter, J.; Chen, B.; Klein, M. L.; Mohamed, F. et al. Liquid Water from First Principles: Investigation of Different Sampling Approaches. J. Phys. Chem. B 2004, 108, 12990-12998.

(34) VandeVondele, J.; Mohamed, F.; Krack, M.; Hutter, J.; Sprik, M.; Parrinello, M. The Influence of Temperature and Density Functional Models in Ab Initio Molecular Dynamics Simulation of Liquid Water. J. Chem. Phys. 2005, 122, 014515.

(35) Todorova, T.; Seitsonen, A. P.; Hutter, J.; Kuo, I.-F. W.; Mundy, C. J. Molecular Dynamics Simulation of Liquid Water: Hybrid Density Functionals. J. Phys. Chem. $B$ 2006, 110, 3685-3691.

(36) Lee, H.-S.; Tuckerman, M. E. Structure of Liquid Water at Ambient Temperature from Ab Initio Molecular Dynamics Performed in the Complete Basis Set Limit. J. Chem. Phys. 2006, 125, 154507.

(37) Guidon, M.; Schiffmann, F.; Hutter, J.; VandeVondele, J. Ab-Initio Molecular Dynamics using Hybrid Density Functionals. J. Chem. Phys. 2008, 128, 214104. 
(38) Morrone, J. A.; Car, R. Nuclear Quantum Effects in Water. Phys. Rev. Lett. 2008, 101, 017801.

(39) Lin, I.-C.; Seitsonen, A. P.; Coutinho-Neto, M. D.; Tavernelli, I.; Rothlisberger, U. Importance of Van der Waals Interactions in Liquid Water. J. Phys. Chem. B 2009, 113, 1127-1131.

(40) Zhang, C.; Donadio, D.; Galli, G. First-Principle Analysis of the IR Stretching Band of Liquid Water. J. Phys. Chem. Lett. 2010, 1, 1398-1402.

(41) Jonchiere, R.; Seitsonen, A. P.; Ferlat, G.; Saitta, A. M.; Vuilleumier, R. Van der Waals effects in ab initio water at ambient and supercritical conditions. J. Chem. Phys. 2011, 135.

(42) Zhang, C.; Wu, J.; Galli, G.; Gygi, F. Structural and Vibrational Properties of Liquid Water from van der Waals Density Functionals. J. Chem. Theory Comput. 2011, 7, 3054-3061.

(43) Zhang, C.; Donadio, D.; Gygi, F.; Galli, G. First Principles Simulations of the Infrared Spectrum of Liquid Water Using Hybrid Density Functionals. J. Chem. Theory Comput. 2011, 7, 1443-1449.

(44) Møgelhøj, A.; Kelkkanen, A. K.; Wikfeldt, K. T.; Schiøtz, J.; Mortensen, J. J.; Pettersson, L. G. M.; Lundqvist, B. I.; Jacobsen, K. W.; Nilsson, A.; Nørskov, J. K. Ab Initio van der Waals Interactions in Simulations of Water Alter Structure from Mainly Tetrahedral to High-Density-Like. J. Phys. Chem. B 2011, 115, 14149-14160.

(45) Laage, D.; Stirnemann, G.; Sterpone, F.; Hynes, J. T. Water Jump Reorientation: From Theoretical Prediction to Experimental Observation. Acc. Chem. Res. 2011, $45,53-62$. 
(46) Heyden, M.; Sun, J.; Forbert, H.; Mathias, G.; Havenith, M.; Marx, D. Understanding the Origins of Dipolar Couplings and Correlated Motion in the Vibrational Spectrum of Water. J. Phys. Chem. Lett. 2012, 2135-2140.

(47) Ceriotti, M.; Cuny, J.; Parrinello, M.; Manolopoulos, D. E. Nuclear Quantum Effects and Hydrogen Bond Fluctuations in Water. Proc. Natl. Acad. Sci. U. S. A. 2013, 110, $15591-15596$.

(48) Kühne, T. D.; Khaliullin, R. Z. Electronic Signature of the Instantaneous Asymmetry in the First Coordination Shell of Liquid Water. Nat Commun 2013, 4, 1450.

(49) Hassanali, A. A.; Cuny, J.; Verdolino, V.; Parrinello, M. Aqueous Solutions: State of the Art in Ab Initio Molecular Dynamics. Philos. Trans. R. Soc. London, Ser. A 2014, 372.

(50) Fritsch, S.; Potestio, R.; Donadio, D.; Kremer, K. Nuclear Quantum Effects in Water: A Multiscale Study. J. Chem. Theory Comput. 2014, 10, 816-824.

(51) DiStasio, R. A.; Santra, B.; Li, Z.; Wu, X.; Car, R. The Individual and Collective Effects of Exact Exchange and Dispersion Interactions on the Ab Initio Structure of Liquid Water. J. Chem. Phys. 2014, 141, 084502.

(52) Gasparotto, P.; Hassanali, A. A.; Ceriotti, M. Probing Defects and Correlations in the Hydrogen-Bond Network of ab Initio Water. J. Chem. Theory Comput. 2016, 12, 1953-1964.

(53) Chen, M.; Ko, H.-Y.; Remsing, R. C.; Calegari Andrade, M. F.; Santra, B.; Sun, Z.; Selloni, A.; Car, R.; Klein, M. L.; Perdew, J. P. et al. Ab Initio Theory and Modeling of Water. Proc. Natl. Acad. Sci. U. S. A. 2017, 114, 10846-10851.

(54) Marsalek, O.; Markland, T. E. Ab initio Molecular Dynamics with Nuclear Quantum 
Effects at Classical Cost: Ring Polymer Contraction for Density Functional Theory. J. Chem. Phys. 2016, 144, 054112.

(55) Speight, J. G. In Catalysis on the Energy Scene; Kaliaguine, S., Mahay, A., Eds.; Studies in Surface Science and Catalysis; Elsevier, 1984; Vol. 19; pp 515-527.

(56) Narten, A. H. X-Ray Diffraction Pattern and Models of Liquid Benzene. J. Chem. Phys. 1977, 67, 2102-2108.

(57) Misawa, M.; Fukunaga, T. Structure of Liquid Benzene and Naphthalene Studied by Pulsed Neutron Total Scattering. J. Chem. Phys. 1990, 93, 3495-3502.

(58) Jorgensen, W. L.; Severance, D. L. Aromatic-Aromatic Interactions: Free Energy Profiles for the Benzene Dimer in Water, Chloroform, and Liquid Benzene. J. Am. Chem. Soc. 1990, 112, 4768-4774.

(59) Cabaço, M. I.; Danten, Y.; Besnard, M.; Guissani, Y.; Guillot, B. Neutron Diffraction and Molecular Dynamics Study of Liquid Benzene and Its Fluorinated Derivatives as a Function of Temperature. J. Phys. Chem. B 1997, 101, 6977-6987.

(60) Tassaing, T.; Cabaço, M. I.; Danten, Y.; Besnard, M. The Structure of Liquid and Supercritical Benzene as Studied by Neutron Diffraction and Molecular Dynamics. J. Chem. Phys. 2000, 113, 3757-3765.

(61) Chelli, R.; Cardini, G.; Procacci, P.; Righini, R.; Califano, S.; Albrecht, A. Simulated Structure, Dynamics, and Vibrational Spectra of Liquid Benzene. J. Chem. Phys. 2000, 113, 6851-6863.

(62) Chelli, R.; Cardini, G.; Ricci, M.; Bartolini, P.; Righini, R.; Califano, S. The Fast Dynamics of Benzene in the Liquid Phase. Part II. A Molecular Dynamics Simulation. Phys. Chem. Chem. Phys. 2001, 3, 2803-2810. 
(63) de la Peña, L. H.; van Zon, R.; Schofield, J.; Opps, S. B. Discontinuous Molecular Dynamics for Rigid Bodies: Applications. J. Chem. Phys. 2007, 126, 074106.

(64) Trumpakaj, Z.; Linde, B. B. Molecular Dynamics Simulation of Benzene. J. Mol. Struct. 2016, 1107, $231-241$.

(65) Porezag, D.; Frauenheim, T.; Köhler, T.; Seifert, G.; Kaschner, R. Construction of Tight-Binding-Like Potentials on the Basis of Density-Functional Theory: Application to Carbon. Phys. Rev. B 1995, 51, 12947-12957.

(66) Elstner, M.; Porezag, D.; Jungnickel, G.; Elsner, J.; Haugk, M.; Frauenheim, T.; Suhai, S.; Seifert, G. Self-Consistent-Charge Density-Functional Tight-Binding Method for Simulations of Complex Material Properties. Phys. Rev. B 1998, 58, $7260-7268$.

(67) Frauenheim, T.; Seifert, G.; Elsterner, M.; Hajnal, Z.; Jungnickel, G.; Porezag, D.; Suhai, S.; Scholz, R. A Self-Consistent Charge Density-Functional Based TightBinding Method for Predictive Materials Simulations in Physics, Chemistry and Biology. Phys. Stat. Solidi (b) 2000, 217, 41-62.

(68) Elstner, M. The SCC-DFTB Method and its Application to Biological Systems. Theo. Chem. Acc. 2006, 116, 316-325.

(69) Maupin, C. M.; Aradi, B.; Voth, G. A. The Self-Consistent Charge Density Functional Tight Binding Method Applied to Liquid Water and the Hydrated Excess Proton: Benchmark Simulations. J. Phys. Chem. B 2010, 114, 6922-6931.

(70) Goyal, P.; Elstner, M.; Cui, Q. Application of the SCC-DFTB Method to Neutral and Protonated Water Clusters and Bulk Water. J. Phys. Chem. B 2011, 115, 6790-6805.

(71) Goyal, P.; Qian, H.-J.; Irle, S.; Lu, X.; Roston, D.; Mori, T.; Elstner, M.; Cui, Q. Molecular Simulation of Water and Hydration Effects in Different Environments: 
Challenges and Developments for DFTB Based Models. J. Phys. Chem. B 2014, $118,11007-11027$.

(72) Lourenço, M. P.; dos Santos, E. C.; Pettersson, L. G. M.; Duarte, H. A. Accurate SCC-DFTB Parametrization for Bulk Water. J. Chem. Theory Comput. 2020, 16, 1768-1778, PMID: 32040315.

(73) Addicoat, M. A.; Stefanovic, R.; Webber, G. B.; Atkin, R.; Page, A. J. Assessment of the Density Functional Tight Binding Method for Protic Ionic Liquids. J. Chem. Theory Comput. 2014, 10, 4633-4643.

(74) Li, J.; Zhu, T.; Cramer, C.; Truhlar, D. New Class IV Charge Model for Extracting Accurate Partial Charges from Wave Functions. J. Phys. Chem. A 1998, 102, 18201831.

(75) Thompson, J. D.; Cramer, C. J.; Truhlar, D. G. Parameterization of Charge Model 3 for AM1, PM3, BLYP, and B3LYP. J. Comput. Chem. 2003, 24, 1291-1304.

(76) Rapacioli, M.; Spiegelman, F.; Talbi, D.; Mineva, T.; Goursot, A.; Heine, T.; Seifert, G. Correction for Dispersion and Coulombic Interactions in Molecular Clusters with Density Functional Derived Methods: Application to Polycyclic Aromatic Hydrocarbon Clusters. J. Chem. Phys. 2009, 130, 244304-10.

(77) Kalinowski, J. A.; Lesyng, B.; Thompson, J. D.; Cramer, C. J.; Truhlar, D. G. Class IV Charge Model for the Self-Consistent Charge Density-Functional Tight-Binding Method. J. Phys. Chem. A 2004, 108, 2545-2549.

(78) Dontot, L.; Spiegelman, F.; Rapacioli, M. Structures and Energetics of Neutral and Cationic Pyrene Clusters. J. Phys. Chem. A 2019, 123, 9531-9543.

(79) Zamith, S.; Ji, M.-C.; L'Hermite, J.-M.; Joblin, C.; Dontot, L.; Rapacioli, M.; Spiegelman, F. Thermal Evaporation of Pyrene Clusters. J. Chem. Phys. 2019, 151, 194303. 
(80) Joblin, C.; Dontot, L.; Garcia, G. A.; Spiegelman, F.; Rapacioli, M.; Nahon, L.; Parneix, P.; Pino, T.; Bréchignac, P. Size Effect in the Ionization Energy of PAH Clusters. The Journal of Physical Chemistry Letters 2017, 8, 3697-3702.

(81) Simon, A.; Rapacioli, M.; Mascetti, J.; Spiegelman, F. Vibrational Spectroscopy and Molecular Dynamics of Water Monomers and Dimers Adsorbed on Polycyclic Aromatic Hydrocarbons. Phys. Chem. Chem. Phys. 2012, 14, 6771-6786.

(82) Simon, A.; Spiegelman, F. Water Clusters Adsorbed on Polycyclic Aromatic Hydrocarbons: Energetics and Conformational Dynamics. J. Chem. Phys. 2013, 138, 194309.

(83) Simon, A.; Spiegelman, F. Conformational Dynamics and Finite-Temperature Infrared Spectra of the Water Octamer Adsorbed on Coronene. Comp. Theor. Chem. 2013, 1021, 54-61.

(84) Oliveira, L. F. L.; Cuny, J.; Moriniere, M.; Dontot, L.; Simon, A.; Spiegelman, F.; Rapacioli, M. Phase Changes of the Water Hexamer and Octamer in the Gas Phase and Adsorbed on Polycyclic Aromatic Hydrocarbons. Phys. Chem. Chem. Phys. 2015, 17, 17079-17089.

(85) Korchagina, K. A.; Simon, A.; Rapacioli, M.; Spiegelman, F.; Cuny, J. Structural Characterization of Sulfur-Containing Water Clusters Using a Density-Functional Based Tight-Binding Approach. J. Phys. Chem. A 2016, 120, 9089-9100.

(86) Heine, T.; Rapacioli, M.; Patchkovskii, S.; Frenzel, J.; Koster, A.; Calaminici, P.; Duarte, H. A.; Escalante, S.; Flores-Moreno, R.; Goursot, A. et al. deMon-Nano Experiment 2009, http://demon-nano.ups-tlse.fr/.

(87) Ceriotti, M.; More, J.; Manolopoulos, D. E. i-PI: A Python Interface for Ab Initio Path Integral Molecular Dynamics Simulations. Comput. Phys. Commun. 2014, 185, 1019-1026. 
(88) Marx, D.; Parrinello, M. Ab Initio Path-Integral Molecular Dynamics. Zeitschrift fur Physik B Condensed Matter 1994, 95, 143-144.

(89) Marx, D.; Parrinello, M. Ab Initio Path Integral Molecular Dynamics: Basic Ideas. J. Chem. Phys. 1996, 104, 4077-4082.

(90) Habershon, S.; Braams, B. J.; Manolopoulos, D. E. Quantum Mechanical Correlation Functions, Maximum Entropy Analytic Continuation, and Ring Polymer Molecular Dynamics. J. Chem. Phys. 2007, 127, 174108-16.

(91) Habershon, S.; Manolopoulos, D. E.; Markland, T. E.; III, T. F. M. Ring-Polymer Molecular Dynamics: Quantum Effects in Chemical Dynamics from Classical Trajectories in an Extended Phase Space. Annu. Rev. Phys. Chem. 2013, 64, 387-413.

(92) Choudhary, A.; Chandra, A. Dynamics of Water in Conical Solvation Shells around a Benzene Solute under Different Thermodynamic Conditions. Phys. Chem. Chem. Phys. 2018, 20, 18328-18339.

(93) Choudhary, A.; Chandra, A. Anisotropic Structure and Dynamics of the Solvation Shell of a Benzene Solute in Liquid Water from Ab Initio Molecular Dynamics Simulations. Phys. Chem. Chem. Phys. 2016, 18, 6132-6145.

(94) Michoulier, E.; Ben Amor, N.; Rapacioli, M.; Noble, J. A.; Mascetti, J.; Toubin, C.; Simon, A. Theoretical Determination of Adsorption and Ionisation Energies of Polycyclic Aromatic Hydrocarbons on Water Ice. Phys. Chem. Chem. Phys. 2018, 20, $11941-11953$.

(95) Simon, A.; Rapacioli, M.; Michoulier, E.; Zheng, L.; Korchagina, K.; Cuny, J. Contribution of the Density-Functional-Based Tight-Binding Scheme to the Description of Water Clusters: Methods, Applications and Extension to Bulk Systems. Mol. Simul. 2019, 45, 249-268. 
(96) Cuny, J.; Korchagina, K.; Menakbi, C.; Mineva, T. Metadynamics Combined with Auxiliary Density Functional and Density Functional Tight-Binding Methods: Alanine Dipeptide as a Case Study. J. Mol. Mod. 2017, 23, 72.

(97) Wu, Q.; Yang, W. Empirical Correction to Density Functional Theory for Van der Waals Interactions. J. Chem. Phys. 2001, 116, 515-524.

(98) Nosé, S. A Unified Formulation of the Constant Temperature Molecular Dynamics Methods. J. Chem. Phys. 1984, 81, 511.

(99) Hoover, W. G. Canonical Dynamics: Equilibrium Phase-Space Distributions. Phys. Rev. A 1985, 31, 1695-1697.

(100) Martyna, G. J.; Klein, M. L.; Tuckerman, M. Nosé-Hoover Chains: The canonical Ensemble via Continuous Dynamics. J. Chem. Phys. 1992, 97, 2635-2644.

(101) Bussi, G.; Donadio, D.; Parrinello, M. Canonical Sampling Through Velocity Rescaling. J. Chem. Phys. 2007, 126, 014101.

(102) Alinchenko, M. G.; Anikeenko, A. V.; Medvedev, N. N.; Voloshin, V. P.; Mezei, M.; Jedlovszky, P. Morphology of Voids in Molecular Systems. A Voronoi-Delaunay Analysis of a Simulated DMPC Membrane. J. Phys. Chem. B 2004, 108, 19056-19067.

(103) Medvedev, N. N.; Voloshin, V. P.; Luchnikov, V. A.; Gavrilova, M. L. An Algorithm for Three-Dimensional Voronoi S-network. J. Comput. Chem. 2006, 27, 1676-1692, The VNP code, http://str.kinetics.nsc.ru/mds/?Software:VNP.

(104) Roux, S. L.; Jund, P. Ring Statistics Analysis of Topological Networks: New Approach and Application to Amorphous GeS2 and SiO2 Systems. Comput. Mater. Sci. 2010, 49, 70-83.

(105) Sosso, G. C.; Caravati, S.; Rotskoff, G.; Vaikuntanathan, S.; Hassanali, A. On the 
Role of Nonspherical Cavities in Short Length-Scale Density Fluctuations in Water. J. Phys. Chem. A 2017, 121, 370-380.

(106) Ansari, N.; Dandekar, R.; Caravati, S.; Sosso, G.; Hassanali, A. High and Low Density Patches in Simulated Liquid Water. J. Chem. Phys. 2018, 149, 204507.

(107) Horn, H. W.; Swope, W. C.; Pitera, J. W.; Madura, J. D.; Dick, T. J.; Hura, G. L.; Head-Gordon, T. Development of an Improved Four-Site Water Model for Biomolecular Simulations: TIP4P-Ew. J. Chem. Phys. 2004, 120, 9665-9678.

(108) Abraham, M. J.; Murtola, T.; Schulz, R.; Páll, S.; Smith, J. C.; Hess, B.; Lindahl, E. GROMACS: High Performance Molecular Simulations through Multi-Level parallelism from Laptops to Supercomputers. SoftwareX 2015, 1-2, 19-25.

(109) Parrinello, M.; Rahman, A. Polymorphic Transitions in Single Crystals: A New Molecular Dynamics Method. J. Appl. Phys. 1981, 52, 7182-7190.

(110) Ceriotti, M.; Bussi, G.; Parrinello, M. Nuclear Quantum Effects in Solids Using a Colored-Noise Thermostat. Phys. Rev. Lett. 2009, 103, 030603.

(111) Ceriotti, M.; Manolopoulos, D. E. Efficient First-Principles Calculation of the Quantum Kinetic Energy and Momentum Distribution of Nuclei. Phys. Rev. Lett. 2012, 109, 100604.

(112) Ceriotti, M.; Parrinello, M.; Markland, T. E.; Manolopoulos, D. E. Efficient Stochastic Thermostatting of Path Integral Molecular Dynamics. J. Chem. Phys. 2010, 133, 124104.

(113) Choi, T. H.; Liang, R.; Maupin, C. M.; Voth, G. A. Application of the SCC-DFTB Method to Hydroxide Water Clusters and Aqueous Hydroxide Solutions. J. Phys. Chem. B 2013, 117, 5165-5179. 
(114) Hamprecht, F. A.; Cohen, A. J.; Tozer, D. J.; Handy, N. C. Development and Assessment of New Exchange-Correlation Functionals. J. Chem. Phys. 1998, 109, 6264-6271.

(115) Mills, R. Self-Diffusion in Normal and Heavy Water in the Range 1-45 deg. J. Phys. Chem. 1973, $77,685-688$.

(116) Doemer, M.; Liberatore, E.; Knaup, J. M.; Tavernelli, I.; Rothlisberger, U. In SituParameterisation of SCC-DFTB Repulsive Potentials by Iterative Boltzmann Inversion. Mol. Phys. 2013, 111, 3595-3607.

(117) Dontot, L.; Suaud, N.; Rapacioli, M.; Spiegelman, F. An Extended DFTB-CI Model for Charge-Transfer Excited States in Cationic Molecular clusters: Model Studies versus Ab Initio Calculations in Small PAH Clusters. Phys. Chem. Chem. Phys. 2016, $18,3545-3557$.

(118) Soper, A. K.; Benmore, C. J. Quantum Differences between Heavy and Light Water. Phys. Rev. Lett. 2008, 101, 065502.

(119) Skinner, L. B.; Huang, C.; Schlesinger, D.; Pettersson, L. G. M.; Nilsson, A.; Benmore, C. J. Benchmark Oxygen-Oxygen Pair-Distribution Function of Ambient Water from X-Ray Diffraction Measurements with a Wide Q-Range. J. Chem. Phys. 2013, $138,074506$.

(120) Wikfeldt, K. T.; Leetmaa, M.; Ljungberg, M. P.; Nilsson, A.; Pettersson, L. G. M. On the Range of Water Structure Models Compatible with X-ray and Neutron Diffraction Data. J. Phys. Chem. B 2009, 113, 6246-6255, PMID: 19358575.

(121) Contreras-Camacho, R. O.; Ungerer, P.; Boutin, A.; Mackie, A. D. Optimized Intermolecular Potential for Aromatic Hydrocarbons Based on Anisotropic United Atoms. 1. Benzene. J. Phys. Chem. B 2004, 108, 14109-14114. 
(122) Falcone, D. R.; Douglass, D. C.; McCall, D. W. Self-Diffusion in Benzene. J. Phys. Chem. 1967, 71, 2754-2755.

(123) Noble, J. A.; Jouvet, C.; Aupetit, C.; Moudens, A.; Mascetti, J. Efficient Photochemistry of Coronene:Water Complexes. Astron. Astrophys. 2017, 599, A124. 


\section{Graphical TOC Entry}

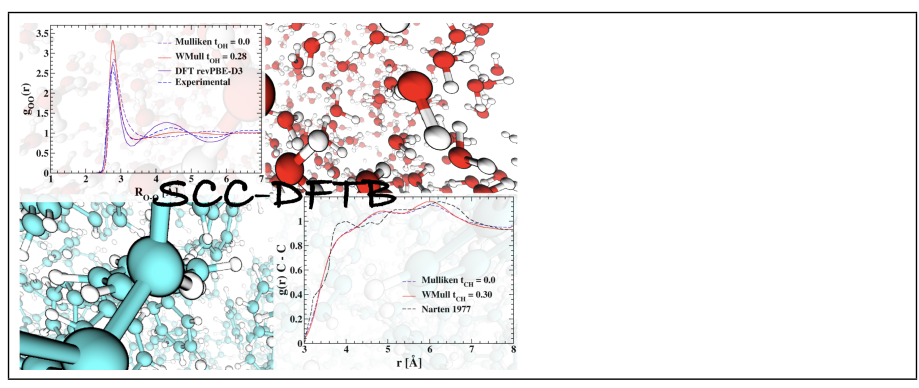

